\title{
Turing patterns in three dimensions
}

\author{
$\operatorname{AUTHOR}(\mathrm{S})$ :
}

Shoji, H; Yamada, K; Ueyama, D; Ohta, T

\section{CITATION:}

Shoji, H ...[et al]. Turing patterns in three dimensions. Physical Review E 2007, 75(4): 046212.

ISSUE DATE:

2007-04

URL:

http://hdl.handle.net/2433/49964

RIGHT:

Copyright 2007 American Physical Society 
PHYSICAL REVIEW E 75, 046212 (2007)

\title{
Turing patterns in three dimensions
}

\author{
Hiroto Shoji ${ }^{1,2, *}$ Kohtaro Yamada, ${ }^{1}$ Daishin Ueyama, ${ }^{2}$ and Takao Ohta ${ }^{1}$ \\ ${ }^{1}$ Department of Physics, Kyoto University, Kyoto, 606-8502, Japan \\ ${ }^{2}$ Meiji Institute for Mathematical Sciences, Meiji University, Higashi Mita, Tama-ku Kawasaki, 214-8571, Japan
}

(Received 30 November 2006; published 24 April 2007)

\begin{abstract}
We investigate three-dimensional Turing patterns in two-component reaction diffusion systems. The FitzHugh-Nagumo equation, the Brusselator, and the Gray-Scott model are solved numerically in three dimensions. Several interconnected structures of domains as well as lamellar, hexagonal, and spherical domains are obtained as stable motionless equilibrium patterns. The relative stability of these structures is studied analytically based on the reduction approximation. The relation with the microphase-separated structures in block copolymers is also discussed.
\end{abstract}

DOI: 10.1103/PhysRevE.75.046212

PACS number(s): 89.75.Kd, 47.54.-r, 05.70.Ln

\section{INTRODUCTION}

Various orders are self-organized far from thermal equilibrium. The theoretical procedures and notions to describe the dynamics of pattern formation have been developed for the last three decades [1-4]. Attempts have also been made to understand morphological orders in biology [5]. Clarification of the mechanisms of the formation of orders and the relationship among them has been one of the fundamental problems in nonequilibrium statistical physics.

Among a number of pattern formation phenomena ranging from crystal growth to hydrodynamic systems, the socalled reaction diffusion equations have been used for many years for modeling a self-organized pattern far from equilibrium. In 1952 Turing showed that a motionless spatially periodic solution can be stable in a coupled reaction diffusion equation with two components if certain conditions are satisfied in the diffusion coefficients and the nonlinearity [6]. This was a surprise because diffusion generally makes the concentration variation uniform in space. The instability found by Turing is now called a diffusion-driven instability or a Turing instability.

It took about 40 years to observe the Turing pattern in real experiments. In a seminal paper, De Kepper and co-workers realized experimentally, for the first time, a Turing-type chemical pattern in a variant of chlorite-iodide reaction in a gel reactor [7]. They realized, by changing the concentration of chemical components, spontaneous breaking of the translational symmetry associated with the formation of a Turing structure [8]. Soon after, Ouyang and Swinney carried out experiments with the same reaction and a reactor more extended in the third direction to produce a stripe pattern and a hexagonal pattern depending on the concentrations of the chemical species $[9,10]$.

It is mentioned here that both the Bordeaux [11] and Austin [12] groups observed a three-dimensional structure and attempted to resolve the symmetry. In fact, De Kepper and co-workers claimed that the pattern which they observed has a body-centered quadratic symmetry $[7,11]$. This was consistent with the previous theoretical analysis of the Brusselator

*Electronic address: hshoji@math.meiji.ac.jp
[13] in an extended system [14]. Identification of the threedimensional symmetry was tried later experimentally in a more elaborated way [15] and by numerical simulations [16]. However, this is a difficult problem because the feeding of the reactor causes the concentration nonuniformity which prevents it from being a truly three-dimensional bulk system. The way to circumvent this difficulty is to employ a closed batch where, however, Turing patterns may appear only transiently [17].

Theoretically, Turing patterns have been studied numerically and analytically as an example of dissipative structures [1]. However, almost all of the previous investigations were restricted to one or two dimensions where only stripe patterns, hexagonal patterns, and labyrinthine patterns can be realized. Only in some special situations can some unusual patterns appear. For example, the coupling between the different spatial modes in bistable systems causes resonant rhombic or quasiperiodic structures as has been predicted by the amplitude equations constructed by a symmetry argument [18]. Spatial resonance patterns such as black eyes and white eyes occur also due to the interaction between different Turing modes [19].

One may think of several reasons as to why threedimensional Turing patterns have been less interested in so far. Three-dimensional numerical simulations of reaction diffusion systems are technically demanded and time consuming. It is also likely that three-dimensional Turing patterns were not so attractive because many researchers thought that two-dimensional patterns might be sufficient to understand the general properties of dissipative structures.

Only a few research groups were interested in Turing structures in three dimensions. Apart from the earlier reference mentioned above [14], De Wit et al. [20] studied Turing instability of the Brusselator in three dimensions by computer simulations. Leppanen et al. [21] as well as De Wit et al. [22] found spherical domains having a bec structure, hexagonally packed cylinders, and lamellar and distorted lamellar structures. A group-theoretical analysis of threedimensional Turing patterns has also been carried out [23].

The purpose of the present paper is to explore the variety of Turing patterns in three dimensions. We emphasize that there are essentially new structures in three dimensions apart from the extension to three dimensions of the corresponding two-dimensional structures. An example is interconnected 
periodic domains whose surfaces are approximately the minimal surfaces such that the average curvature is equal to zero everywhere [24]. This kind of patterns can never exist in two dimensions. The possibility of such patterns in three dimensions was mentioned in Ref. [22]. A preliminary version of our study has been published in Ref. [25] where the results of the FitzHugh-Nagumo equation [26] have been reported. In the present paper, we shall describe further the details of the interconnected Turing patterns in the FitzHughNagumo equation. In order to demonstrate that these interconnected structures are not restricted to the FitzHughNagumo equation, we show the results of the Brusselator and the Gray-Scott model [27]. Those two are also typical reaction diffusion systems having been studied for many years.

The present paper is organized as follows. In Sec. II, we carry out numerical simulations of the FitzHugh-Nagumo reaction diffusion equation. Since the system size used in the simulations is set to be comparable to the spatial period of Turing patterns, we change the system size to find the most commensurate size and to avoid any artifacts due to the finiteness of the system size. In order to compare the relative stability of the patterns obtained, we make a reduction of the FitzHugh-Nagumo equation such that it possesses approximately a Lyapunov functional. In Sec. III, we investigate the Turing patterns in the Brusselator. In contrast to previous studies [20,22], we shall explore the Turing structures near the bifuraction threshold. We show in Sec. IV that the interconnected structures obtained in the FitzHugh-Nagumo equation and the Brusselator appear also in the Gray-Scott model. Finally, the results are discussed in Sec. V. In particular, the similarity to and the difference from microphase separation in polymeric systems are discussed. A reduced equation for the Brusselator is derived in the Appendix.

\section{FITZHUGH-NAGUMO EQUATION}

\section{A. Model and numerical simulations}

The FitzHugh-Nagumo equation is given by the coupled reaction diffusion equations [26]

$$
\begin{gathered}
\frac{\partial u}{\partial t}=D_{u} \nabla^{2} u+u-u^{3}-v, \\
\frac{\partial v}{\partial t}=D_{v} \nabla^{2} v+\gamma(u-\alpha v-\beta),
\end{gathered}
$$

where the constants $\alpha, \beta, \gamma, D_{u}$, and $D_{v}$ are all positive. This set of equations has been introduced as a model equation of impulse propagation along the nerve axion [28]. In its original version, the diffusion term of the variable $v$ was absent and only the $\gamma u$ term existed in Eq. (2). By adding the diffusion term to the coefficient $D_{v}$, the set of equations (1) and (2) are the model equations for the Belousov-Zhabotinsky chemical reaction [29].

Here we add two more previous studies which might be useful for further developments. One is the analysis without diffusion terms, which is a powerful method of finding experimentally new chemical oscillators [30]. The other is a microscopic modeling of the FitzHugh-Nagumo equation by
Malevanets and Kapral, which will be relevant to possible Turing patterns in nanoscopic or mesoscopic scales [31]. Furthermore, they have studied three-dimensional objects such as a Hopf link and a figure-8 knot.

Equations (1) and (2) have a time-independent uniform solution $(\bar{u}, \bar{v})$ which are defined through $\bar{u}-\bar{u}^{3}-\bar{v}=0$ and $\bar{u}-\alpha \bar{v}-\beta=0$. We are concerned with the monostable situation when the diffusions are absent. The bistable case has been studied in terms of the amplitude equations in two dimensions $[18,32]$. The linear stability analysis of the uniform solution is readily carried out. Put $(u-\bar{u}, v-\bar{v}) \sim \exp (i k x$ $+\lambda t)$. The eigenvalue $\lambda$ is a solution of the algebraic equation

$$
\begin{aligned}
\lambda^{2}+ & \left\{\left(D_{u}+D_{v}\right) k^{2}-\left(3 \bar{u}^{2}+\alpha \gamma-1\right)\right\} \lambda+\left(D_{u} k^{2}+3 \bar{u}^{2}-1\right) \\
& \times\left(D_{v} k^{2}+\alpha \gamma\right)+\gamma=0 .
\end{aligned}
$$

The uniform solution $(\bar{u}, \bar{v})$ becomes unstable when the real part of $\lambda$ is positive. (Actually we consider the situation that the eigenvalue is always real.) The eigenvalue becomes positive first at the critical wave number given by

$$
k_{c}^{2}=\frac{1-3 \bar{u}^{2}}{2 D_{u}}-\frac{\alpha \gamma}{2 D_{v}} .
$$

The parameter region where $(\bar{u}, \bar{v})$ is linearly unstable is given by the condition

$$
\frac{\left\{D_{v}\left(1-3 \bar{u}^{2}\right)-\alpha \gamma D_{u}\right\}^{2}}{4 D_{u} D_{v}}+\alpha \gamma\left(1-3 \bar{u}^{2}\right)-\gamma>0 .
$$

It is readily shown that the condition $k_{c}^{2}>0$ is satisfied if $D_{u} \ll D_{v}$.

In order to investigate the time evolution in the region given by the inequality (5), we have carried out numerical simulations for the coupled set of equations (1) and (2) in three dimensions. The space is divided into $N^{3}$ cubic cells with cell size $\delta x$. The periodic boundary conditions are imposed at the system boundaries. The choice of cell size $\delta x$ will be described shortly. We utilize the numerical method so-called libfles_fft library [33] which is a fast linear equation solver based on the fast Fourier transform (FFT) algorithm proposed by Eyre [34]. In this method, the time increment $\delta t$ is set as $\delta t=0.2$ throughout the present paper. The forward Euler integration method is also applied to verify that the results are independent of the numerical scheme. In this method, the time increment depends on the cell size $\delta x$ and the model equations. For example, $\delta t=5.0 \times 10^{-4}$ for $L$ $=\delta x N=0.17$ with $N=50$ and $\delta x=3.40 \times 10^{-3}$ in the FitzHugh-Nagumo equation. In order to diminish the anisotropy in discretizing the Laplacian, we employ a 27-point difference scheme [35] in both methods.

One of the most crucial technical problems is to determine the spatial period of the periodic structures. When the system size $L=\delta x N$ is comparable to the spatial period, any small mismatch between the system size and the true period would cause a drastic artifact for the final time-independent solution in the simulations. Although the critical wave number $k_{c}$ at the bifurcation point can be obtained as Eq. (4) by linear stability analysis, analytical evaluation of the true period at the post-threshold is generally impossible because of 


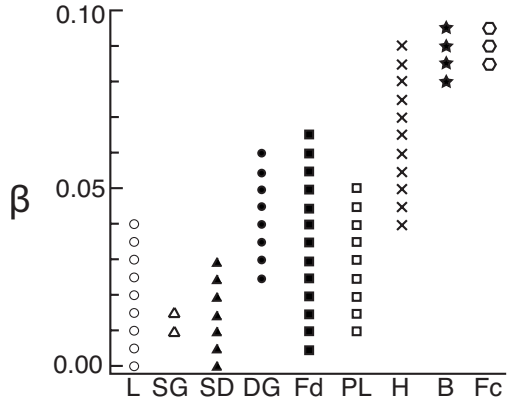

FIG. 1. Nine stationary solutions obtained in the FitzHughNagumo equation. The meanings of L, SG, SD, DG, Fd, PL, H, B, and $\mathrm{Fc}$ are given in the text.

the strong nonlinearity. In order to overcome this difficulty, we change the cell size $\delta x$ in both this section and the subsequent sections. Numerical simulations are performed mainly for $D_{u}=5.0 \times 10^{-5}, D_{v}=5.0 \times 10^{-3}, \alpha=0.5$, and $\gamma$ $=26$ and by varying $\beta$.

We start with the unstable uniform solution $(\bar{u}, \bar{v})$ with small random perturbation superimposed. Thus the initial profiles of $u$ and $v$ are completely random without any spatial correlation. Furthermore, random forces $\xi$ without any space-time correlations are added to Eqs. (1) and (2) independently at each time step and at each cell. We have generated a random real number uniformly distributed between $-\sigma / 2$ and $\sigma / 2$. The strength of the noise $\sigma$ is adjusted in each run such that trapping of the structures at any metastable states is avoided. This procedure is applied not only to the FitzHugh-Nagumo equation but also to the Brusselator and to the Gray-Scott model described below.

The asymptotic stationary solutions obtained for $N=32$ are listed in Fig. 1. The abbreviations L, H, and B mean lamellar, hexagonal, and bcc structures, respectively. Note that three or more different structures appear at the same value of $\beta$. The structures DG, Fd, PL, Fc, SG, and SD will be identified below.

This figure was obtained in the following way. First of all, we emphasize that the values of $\delta x$ and $\sigma$ are not changed during each run. It should be noted that this set of simulations is very systematic and detailed. For example, we have carried out simulations for $\beta=0.04$ starting from five differ-

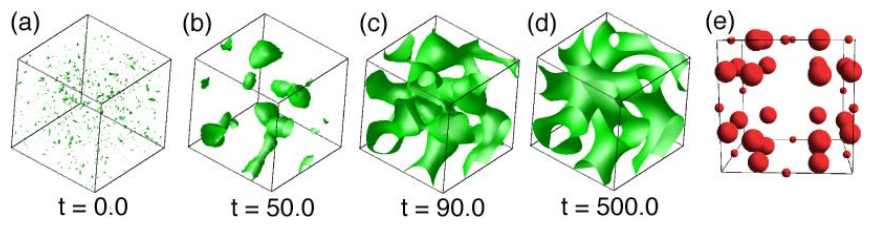

FIG. 2. (Color online) Formation of double-gyroid structure in the FitzHugh-Nagumo equation. (a)-(d) Structural evolution of domains for $\beta=0.04, L=0.170=\delta x N$ with $\delta x=5.32 \times 10^{-3}$ and $N=32$, and $\sigma=1.0 \times 10^{-2}$, and (e) Bragg spots for $I_{k}>1.5 \times 10^{-4}$. For the sake of clarity, the domains in (a) represent the isosurface of $u$ $=0.07949$ whereas those in (b) $-(\mathrm{d})$ represent the isosurface of $u$ $=0.05$. In (e) the Bragg points on the same plane are connected with a line. The radius of the spheres corresponds to the relative peak intensity. In this figure as well as in all the figures below for $I_{k}$, the Bragg point at the origin is not drawn.
TABLE I. Bragg spots and their intensities (arbitrary units) of double-gyroid structure. Here and in all the tables below, the Bragg positions symmetric with respect to the origin are not given.

\begin{tabular}{lccc}
\hline \hline Peak position & $I_{k}$ & Peak position & $I_{k}$ \\
\hline$(2,1,1)$ & 0.009524 & $(1,-1,-2)$ & 0.009515 \\
$(2,1,-1)$ & 0.009520 & $(1,-2,1)$ & 0.009527 \\
$(2,-1,1)$ & 0.009512 & $(1,-2,-1)$ & 0.009506 \\
$(2,-1,-1)$ & 0.009524 & $(2,2,0)$ & 0.001700 \\
$(1,2,1)$ & 0.009527 & $(2,0,2)$ & 0.001702 \\
$(1,2,-1)$ & 0.009512 & $(2,0,-2)$ & 0.001705 \\
$(1,1,2)$ & 0.009519 & $(2,-2,0)$ & 0.001703 \\
$(1,1,-2)$ & 0.009517 & $(0,2,2)$ & 0.001702 \\
$(1,-1,2)$ & 0.009506 & $(0,2,-2)$ & 0.001704 \\
\hline \hline
\end{tabular}

ent random initial conditions and for about 100 values of $L$ $=\delta x N(0.06<L<0.25$ with increment 0.002$)$ and for ten values of $\sigma$ in the interval $5 \times 10^{-4}<\sigma<2 \times 10^{-2}$. This means that there are about $5000 \approx 5 \times 100 \times 10$ independent runs only for one value of $\beta$. Figure 1 indicates that the structures $\mathrm{L}, \mathrm{DG}, \mathrm{Fd}, \mathrm{PL}$, and $\mathrm{H}$ occurred in these runs. Similar runs are repeated for other values of $\beta$ to complete Fig. 1 .

Before closing this section, we comment about the system size $N$. In order to verify that the results in Fig. 1 are insensitive to $N$, we have carried out similar simulations for $N$ $=50$ by the forward Euler method and for $N=64$ by the libfles_fft library method. We emphasize that the results in Fig. 1 have been reproduced completely both for $N=50$ and $N=64$. Therefore, hereafter, we shall show the results only for $N=32$ and present explicitly the values of $\delta x$ and $\sigma$.

\section{B. Motionless periodic patterns}

The time evolution of the concentration for $\beta=0.04$ starting from the random initial configuration is displayed in Fig. 2. The asymptotic pattern DG is shown in Fig. 2(d). We have carried out a Fourier transformation of the asymptotic value of $u$ and obtained the Bragg peaks which are listed in Table I together with the peak intensities. The peak intensity is defined by $I_{k}=\left|u_{k}\right|^{2}$ where $u_{k}$ is the Fourier transform of $u$. The peak position is defined by $\vec{k} N / 2 \pi$ with $\vec{k}$ the wave vector. The peaks whose intensities are smaller than 5.0 $\times 10^{-6}$ are not shown in Table I.

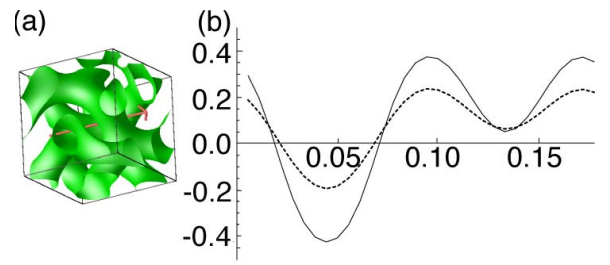

FIG. 3. (Color online) Spatial variation of the concentrations $u$ and $v$ for double-gyroid structure for $\beta=0.05, L=0.176=\delta x N$ with $\delta x=5.53 \times 10^{-3}$ and $N=32$, and $\sigma=5.0 \times 10^{-3}$. (a) The isosurface of $u$ of double-gyroid structure. (b) Concentration of $u$ (solid line) and $v$ (dotted line) along the red arrow in (a). 


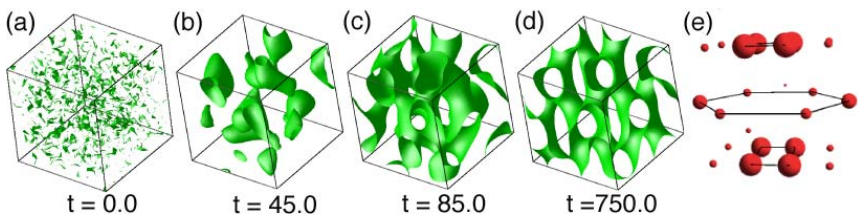

FIG. 4. (Color online) Fddd structure obtained in the FitzHughNagumo equation. (a)-(d) Structural evolution of domains for $\beta$ $=0.04, L=0.176=\delta x N$ with $\delta x=5.50 \times 10^{-3}$ and $N=32$, and $\sigma$ $=5.0 \times 10^{-3}$, and (e) Bragg spots for $I_{k}>1.5 \times 10^{-4}$. The meanings of (e) are the same as those in Fig. 2. In order to make the initial randomness visible the domains in (a) represent the isosurface of $u=0.07949$ whereas those in (b)-(d) represent the isosurface of $u$ $=0.05$.

Figure 3 shows the stationary profile of $u$ and $v$ in the structure DG. The values of the concentrations are taken along the red arrow in Fig. 3(a). Note that the variation is almost sinusoidal without no sharp interface separating the two domains.

One of the well-known interconnected structures is the so-called double-gyroid structure which is represented approximately by the level set equation

$$
\begin{aligned}
0= & 8(1-\eta)[\sin 2 x \sin z \cos y+\sin 2 y \sin x \cos z \\
& +\sin 2 z \sin y \cos x]-4 \eta[\cos 2 x \cos 2 y+\cos 2 y \cos 2 z \\
& +\cos 2 z \cos 2 x]-\xi
\end{aligned}
$$

where $\eta$ and $\xi$ are parameters [36]. The Bragg positions shown in Table I are completely consistent with the reciprocal lattice vectors obtained from Eq. (6). See also Eqs. (12) and (13) below. Therefore we identify the structure DG shown in Fig. 2 with the double-gyroid structure.

Now we turn to the structure Fd in Fig. 1. The domain evolution, the asymptotic structure, and the Bragg positions are shown in Fig. 4. The Bragg peaks together with the peak intensities are given in Table II where the Bragg spots only for $I_{k} \geqslant 10^{-6}$ are presented. These results clearly indicate that the structure Fd should be identified with the Fddd structure. It should be mentioned here that the Fddd structure has been predicted in the microphase separation of diblock copolymer melts $[37,38]$.

The formation of the PL structure is displayed in Fig. 5 together with the Bragg positions. This is a layer structure having holes in each layer and is identified with the so-called

TABLE II. Bragg spots and their intensities (arbitrary units) of Fddd structure.

\begin{tabular}{lccc}
\hline \hline Peak position & $I_{k}$ & Peak position & $I_{k}$ \\
\hline$(2,1,1)$ & 0.026959 & $(2,-2,0)$ & 0.003251 \\
$(1,2,1)$ & 0.026959 & $(0,3,1)$ & 0.000176 \\
$(2,1,-1)$ & 0.026959 & $(3,0,1)$ & 0.000176 \\
$(1,2,-1)$ & 0.026959 & $(3,0,-1)$ & 0.000176 \\
$(1,-1,2)$ & 0.007059 & $(0,3,-1)$ & 0.000176 \\
$(1,-1,-2)$ & 0.007059 & $(0,0,4)$ & 0.000075 \\
\hline \hline
\end{tabular}

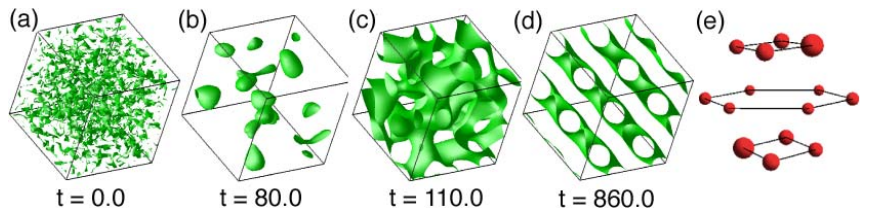

FIG. 5. (Color online) Perforated lamellar structure obtained in the FitzHugh-Nagumo equation. (a)-(d) Structural evolution of domains for $\beta=0.045, L=0.200=\delta x N$ with $\delta x=6.25 \times 10^{-3}$ and $N$ $=32$, and $\sigma=5.0 \times 10^{-3}$, and (e) Bragg spots for $I_{k}>1.5 \times 10^{-4}$. The meanings of (e) are the same as those in Fig. 2. In order to make the initial randomness visible the domains in (a) represent the isosurface of $u=0.08925$ whereas those in (b)-(d) represent the isosurface of $u=0.05$.

perforated lamellar structure. It is noted that the intensity of the higher-order peaks in Table III decreases more slowly than that of the Fddd structure given in Table II. We have found that the holes in each layer take a distorted hexagonal configuration and locate at the same position every two layers $(A B A B$ type). We have not obtained another type of perforated lamellar structure having the holes at the same location every three layers $(A B C A B C$ type).

There are two other interconnected periodic structures in Fig. 1. SD means the single-diamond structure, and SG means the single-gyroid structure. Since these structures appear also in the Brusselator, we shall explain the details in the next section.

Now we describe the structures made of disconnected spherical domains. Even in this case, a nontrivial phenomenon has been obtained. Figure 6 shows the time evolution of the structure B. The Bragg spots in Table IV clearly indicate that this has a bcc symmetry. On the other hand, Fig. 7 displays the pattern formation and its Bragg positions of the structure Fc. See also Table V. It is evident that this structure is an FCC (face-centered-cubic) structure. As seen in Fig. 1, bcc and fcc structures are obtained for the same values of the parameter $\beta$ except for $\beta=0.08$. The formation of either bcc structure or fcc structure depends on the cell size. Actually the bcc structure for $\beta=0.09$ in Fig. 6 is obtained for $L$ $=0.198=\delta x N$ with $\delta x=6.2 \times 10^{-3}$ and $N=32$, and $\sigma=1.0$ $\times 10^{-3}$ whereas the fcc structure in Fig. 7 is for $L=0.128$ $=\delta x N$ with $\delta x=4.0 \times 10^{-3}$ and $N=32$, and $\sigma=1.0 \times 10^{-3}$.

\section{Reduced system}

As shown in Fig. 1, several structures exist for the same set of parameters. Therefore one of the basic questions is to determine which is the most stable structure. However, this is

TABLE III. Bragg spots and their intensities (arbitrary units) of perforated lamellar structure.

\begin{tabular}{lccc}
\hline \hline Peak position & $I_{k}$ & Peak position & $I_{k}$ \\
\hline$(2,-1,-1)$ & 0.089158 & $(0,2,2)$ & 0.005162 \\
$(2,1,-1)$ & 0.007418 & $(0,2,0)$ & 0.002782 \\
$(2,-1,1)$ & 0.007369 & $(0,0,2)$ & 0.002761 \\
$(2,1,1)$ & 0.006495 & & \\
\hline \hline
\end{tabular}



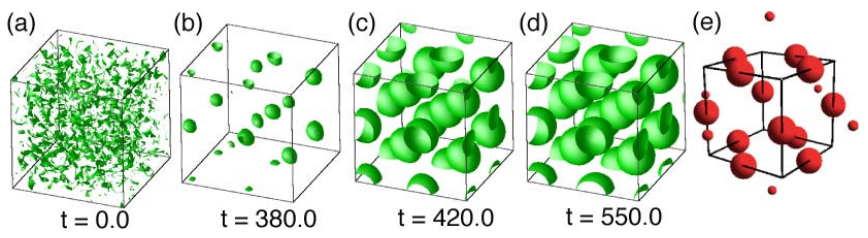

FIG. 6. (Color online) bcc structure obtained in the FitzHughNagumo equation. (a)-(d) Structural evolution of domains for $\beta$ $=0.09, L=0.198=\delta x N$ with $\delta x=6.20 \times 10^{-3}$ and $N=32$, and $\sigma$ $=1.0 \times 10^{-3}$ represented by the isosurface of $u$ and (e) Bragg spots for $I_{k}>10^{-4}$. The meanings of (e) are the same as those in Fig. 2. In order to make the initial randomness visible the domains in (a) represent the isosurface of $u=0.17468$ whereas those in (b)-(d) represent the isosurface of $u=0.05$.

highly nontrivial because Eqs. (1) and (2) are nonvariational with no Lyapunov functional. Hence it is impossible to compare a free-energy-like quantity for the structures as in the thermal equilibrium case. Probably, the most general method to see the relative stability of two states in a nonvariational system like the present model equations is to examine the motion of a planer interface separating those two structures as was attempted in Ref. [21] for a bistable reaction diffusion system. If one of the structures invades the other, one may conclude that the former is more stable than the latter. This can be carried out readily in two dimensions but is not easy in three dimensions because one has to provide a sufficiently large system to see the intrinsic bulk property without suffering from finite-size effects.

One of the remarkable properties of the FitzHughNagumo equations (1) and (2) is that they possesses a Lyapunov functional in a special limit [39]. That is, in the limit $\gamma, D_{v} \rightarrow \infty$ with $\gamma / D_{v}$ finite, one may put $\partial v / \partial t=0$ in Eq. (2), so that the variable $v$ can be eliminated. As a result, we have a closed equation only for $u$,

$$
\frac{\partial u}{\partial t}=-\frac{\delta F}{\delta u}
$$

where

$$
\begin{aligned}
F\{u\}= & \int d \vec{r}\left[\frac{D_{u}}{2}(\vec{\nabla} u)^{2}-\frac{u^{2}}{2}+\frac{u^{4}}{4}\right]+\frac{\gamma}{2 D_{v}} \int d \vec{r} \int d \vec{r}^{\prime} G\left(\vec{r}, \vec{r}^{\prime}\right) \\
& \times[u(\vec{r}, t)-\beta]\left[u\left(\vec{r}^{\prime}, t\right)-\beta\right] .
\end{aligned}
$$

The Green function is defined through

TABLE IV. Bragg spots and their intensities (arbitrary unit) of bec structure.

\begin{tabular}{lccc}
\hline \hline Peak position & $I_{k}$ & Peak position & $I_{k}$ \\
\hline$(2,2,0)$ & 0.013569 & $(0,2,2)$ & 0.013569 \\
$(-2,2,0)$ & 0.013569 & $(0,4,0)$ & 0.000212 \\
$(2,0,2)$ & 0.013569 & $(0,0,4)$ & 0.000212 \\
$(-2,0,2)$ & 0.013569 & $(4,0,0)$ & 0.000212 \\
$(0,-2,2)$ & 0.013569 & & \\
\hline \hline
\end{tabular}

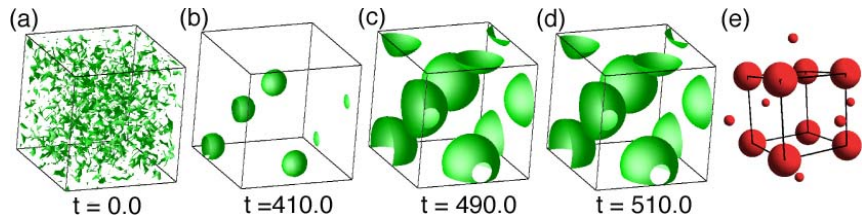

FIG. 7. (Color online) fcc structure obtained in the FitzHughNagumo equation. (a)-(d) Structural evolution of domains for $\beta$ $=0.09, L=0.128=\delta x N$ with $\delta x=4.00 \times 10^{-3}$ and $N=32$, and $\sigma$ $=1.0 \times 10^{-3}$, represented by the isosurface of $u$ and (e) Bragg spots for $I_{k}>10^{-4}$. The meanings of (e) are the same as those in Fig. 2. The values of the isosurface are the same as those in Fig. 6.

$$
\left(-\nabla^{2}+\frac{\alpha \gamma}{D_{v}}\right) G\left(\vec{r}, \vec{r}^{\prime}\right)=\delta\left(\vec{r}-\vec{r}^{\prime}\right) .
$$

Since $\gamma / D_{v}$ is positive, the functional $F$ is bounded below and plays a role of a Lyapunov functional.

Here we make a remark that the Lyapunov functional (8) is the same as the free energy functional in microphase separation of diblock copolymers [40] if $\alpha=0$ and $\beta$ are replaced by the spatial average of $u$. In this case $u$ has the meaning of a local concentration difference between two blocks consisting of each copolymer chain. Since $u$ is a conserved quantity in block copolymers, the time-evolution equation is not given by Eq. (7) but by

$$
\frac{\partial u}{\partial t}=\nabla^{2} \frac{\delta F}{\delta u} .
$$

It is mentioned here that Teramoto and Nishiura [41] have solved numerically Eq. (10) in three dimensions with $N$ $=32$ as in the present study and have obtained double-gyroid and some other structures.

It is also remarked that competition of the short-range interaction arising from the first term in Eq. (8) and a longrange interaction such as the last term in Eq. (8) is essential to get stable periodic structures. In the systems of thermodynamic equilibrium, this has been recognized in the respective case [42].

We investigate the relative stability of Turing structures by using the Lyapunov functional (8) in the reduced system (7). Of course, this is an approximation for the original coupled set of equations (1) and (2). However, we expect that some insights into the stability are provided by this method.

In order to evaluate the functional (8), we employ the modes listed in Tables I-V for each structure except for the

TABLE V. Bragg spots and their intensities (arbitrary unit) of fcc structure.

\begin{tabular}{lccc}
\hline \hline Peak position & $I_{k}$ & Peak position & $I_{k}$ \\
\hline$(1,1,1)$ & 0.011289 & $(0,0,2)$ & 0.003701 \\
$(1,1,-1)$ & 0.011281 & $(0,2,0)$ & 0.003698 \\
$(1,-1,1)$ & 0.011281 & $(2,0,0)$ & 0.003698 \\
$(1,-1,-1)$ & 0.011277 & & \\
\hline \hline
\end{tabular}




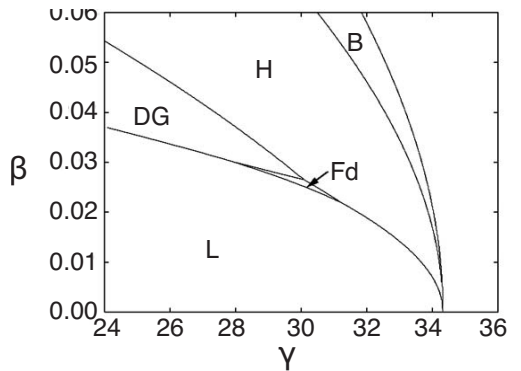

FIG. 8. Phase diagram in the $\gamma-\beta$ plane. The most stable regions for lamellar, hexagonal, double gyroid, Fddd, and bcc structures are indicated by L, H, DG, Fd, and B, respectively.

perforated lamellar structure because the convergence of the mode expansion for this structure is not enough as indicated in Table III. We shall apply another procedure to evaluate the free energy of the perforated lamellar structure.

We employ the mode-expansion method and expand $u$ as follows:

$$
\begin{aligned}
u(\vec{r}, t)= & \bar{u}+\left[\sum_{i=1}^{12} a_{i}(t) e^{i \vec{q}_{i} \cdot \vec{r}}+\sum_{j=1}^{6} b_{j}(t) e^{i \vec{p}_{j} \cdot \vec{r}}+\sum_{l=1}^{12} c_{l}(t) e^{i \overrightarrow{h_{l}} \cdot \vec{r}}\right. \\
& \left.+\sum_{m=1}^{3} d_{m}(t) e^{i \vec{s}_{m} \cdot \vec{r}}+\text { c.c. }\right]
\end{aligned}
$$

where $a_{i}, b_{j}, c_{l}$, and $d_{m}$ are real amplitudes and "c.c." means complex conjugate. The reciprocal vectors $\vec{q}_{i}$ and $\vec{p}_{j}$ are defined as follows:

$$
\begin{gathered}
\vec{q}_{1}=C_{Q}(2,-1,1), \quad \vec{q}_{2}=C_{Q}(-2,1,1), \\
\vec{q}_{3}=C_{Q}(-2,-1,1), \quad \vec{q}_{4}=C_{Q}(2,1,1), \\
\vec{q}_{5}=C_{Q}(-1,-2,1), \quad \vec{q}_{6}=C_{Q}(1,-2,1), \\
\vec{q}_{7}=C_{Q}(-1,2,1), \quad \vec{q}_{8}=C_{Q}(1,2,1), \\
\vec{q}_{9}=C_{Q}(1,-1,-2), \quad \vec{q}_{10}=C_{Q}(1,1,-2), \\
\vec{q}_{11}=C_{Q}(-1,1,-2), \quad \vec{q}_{12}=C_{Q}(-1,-1,-2), \\
\vec{p}_{1}=C_{Q}(2,2,0), \quad \vec{p}_{2}=C_{Q}(2,-2,0), \\
\vec{p}_{3}=C_{Q}(0,2,2), \quad \vec{p}_{4}=C_{Q}(0,-2,2), \\
\vec{p}_{5}=C_{Q}(2,0,2), \quad \vec{p}_{6}=C_{Q}(-2,0,2),
\end{gathered}
$$

where $C_{Q} \equiv Q / \sqrt{6}$ and $Q=\left|\vec{q}_{i}\right|$.

It should be noted that these 18 modes of the doublegyroid structure in Table I cover lamellar, hexagonal, Fddd, and bcc structures as a special case [43] and we have applied this expansion in our previous paper [25]. In the present paper, we add the higher modes $\vec{h}_{l}$ and $\vec{s}_{m}$ defined by

$$
\begin{array}{ll}
\vec{h}_{1}=C_{Q}(1,3,0), & \vec{h}_{2}=C_{Q}(-1,3,0), \\
\vec{h}_{3}=C_{Q}(3,1,0), & \vec{h}_{4}=C_{Q}(-3,1,0),
\end{array}
$$

$$
\begin{gathered}
\vec{h}_{5}=C_{Q}(0,1,3), \quad \vec{h}_{6}=C_{Q}(0,-1,3), \\
\vec{h}_{7}=C_{Q}(0,3,1), \quad \vec{h}_{8}=C_{Q}(0,-3,1), \\
\vec{h}_{9}=C_{Q}(3,0,1), \quad \vec{h}_{10}=C_{Q}(3,0,-1), \\
\vec{h}_{11}=C_{Q}(1,0,3), \quad \vec{h}_{12}=C_{Q}(1,0,-3), \\
\vec{s}_{1}=C_{Q}(4,0,0), \quad \vec{s}_{2}=C_{Q}(0,4,0), \\
\vec{s}_{3}=C_{Q}(0,0,4) .
\end{gathered}
$$

These new modes represent the next higher harmonics of the Fddd structure (see Table II). It is noted that the 33 reciprocal vectors given by Eqs. (12) and (13) are not necessarily independent but satisfy some relations such as

$$
\begin{gathered}
\vec{q}_{1}+\vec{q}_{2}-\vec{q}_{3}-\vec{q}_{4}=0, \quad \vec{q}_{1}-\vec{q}_{3}-\vec{p}_{1}-\vec{p}_{2}=0, \\
\vec{q}_{1}-\vec{h}_{2}-\vec{h}_{9}+\vec{s}_{2}=0, \quad \vec{p}_{1}-\vec{h}_{3}+\vec{h}_{6}+\vec{h}_{12}=0, \\
\vec{q}_{1}+\vec{q}_{7}+\vec{q}_{12}=0, \quad \vec{q}_{1}+\vec{p}_{6}-\vec{h}_{6}=0, \\
\vec{p}_{5}+\vec{p}_{6}-\vec{s}_{3}=0 .
\end{gathered}
$$

Substituting Eq. (11) into Eq. (8) and ignoring the higher harmonics, which is justified when the concentration variation is almost sinusoidal as shown in Fig. 3, the Lyapunov functional (8) can be written in terms of $a_{i}, b_{j}, c_{l}, d_{m}$, and $Q$ as $F_{\text {amp }}=F\left(\left\{a_{i}\right\},\left\{b_{j}\right\},\left\{c_{l}\right\},\left\{d_{m}\right\}, Q\right)$ although we do not write down its whole expression because it is lengthy. The part which depends on $Q$ is given by

$$
\begin{aligned}
F_{n l}= & \left(D_{u} Q^{2}+\frac{2 \gamma}{2 D_{v} Q^{2}+\gamma}\right) \\
& \times \sum_{i=1}^{12} a_{i}^{2}+\left(D_{u} Q_{2}^{2}+\frac{2 \gamma}{2 D_{v} Q_{2}^{2}+\gamma}\right) \\
& \times \sum_{j=1}^{6} b_{j}^{2}+\left(D_{u} Q_{3}^{2}+\frac{2 \gamma}{2 D_{v} Q_{3}^{2}+\gamma}\right) \\
& \times \sum_{l=1}^{12} c_{l}{ }^{2}+\left(D_{u} Q_{4}{ }^{2}+\frac{2 \gamma}{2 D_{v} Q_{4}{ }^{2}+\gamma}\right) \sum_{m=1}^{3} d_{m}{ }^{2},
\end{aligned}
$$

with $Q^{2}=3 Q_{2}{ }^{2} / 4=3 Q_{3}{ }^{2} / 5=3 Q_{4}{ }^{2} / 8$. The magnitude $Q$ is also determined by the condition $\partial F_{n l} / \partial Q=0$.

The fcc structure which is not a subgroup of the doublegyroid structure is treated similarly by using the modes listed in Table V. In this way we can evaluate the Lyapunov functional for each structure.

The phase diagram in Fig. 8 shows the most stable structures on the plane of $\gamma$ and $\beta$ for $D_{u}=5.0 \times 10^{-5}, D_{v}=5.0$ $\times 10^{-3}$, and $\alpha=0.5$. It is emphasized here that the results in Fig. 8 are free from the cell size $\delta x$ or the system size $L$ $=\delta x N$ since we have employed the mode-expansion method. The spatial period of the structure $(2 \pi / Q)$ has been deter- 


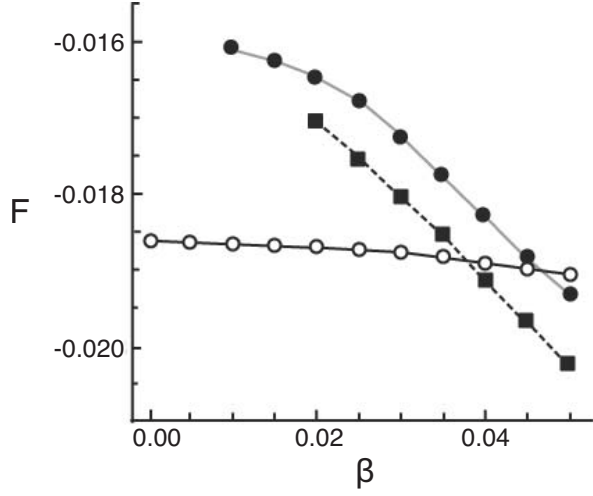

FIG. 9. Values of the Lyapunov functional for lamellar (white circle), double-gyroid (square), and perforated-lamellar (black circle) structures for $\gamma=26.0$. The lines for double-gyroid and perforated-lamellar structures are terminated at some value of $\beta$, beyond which those stable solutions become unstable.

mined uniquely by the minimization of the free energy (Lyapunov function) as mentioned just below Eq. (15). The solid curves in this figure indicate the boundary that the free energies of the two structures on both sides of the line are the same. It is mentioned that there is no regime where the fcc structure is most stable. We note that there is a small but finite region where the Fddd structure is mostly stable. This is a result which was not obtained in our previous paper [25] where we took account only of the 18 modes (12).

Finally we evaluate the Lyapunov functional for the perforated-lamellar structure by substituting the asymptotic values of $u$ directly to Eq. (8). However, this is a timeconsuming procedure because the equilibrium period of the structure should also be determined by optimization of the Lyapunov functional. Remember that the equilibrium wave number for other structures can be evaluated from the condition $\partial F\left(\left\{a_{i}\right\},\left\{b_{j}\right\}, Q\right) / \partial Q=0$. Repeating the numerical simulations for different values of the mesh size $\delta x$ to find the optimal period, we obtain the smallest value of the Lyapunov functional of the perforated-lamellar structure. The result is displayed in Fig. 9 for $D_{u}=5.0 \times 10^{-5}, D_{v}$ $=5.0 \times 10^{-3}, \alpha=0.5$, and $\gamma=26.0$. The values of the Lyapunov functional for the double-gyroid structure and the lamellar structure obtained similarly are also shown for comparison. It is clear that there is no regime that the perforatedlamellar structure has a smaller value of the Lyapunov functional.

So far we have shown the numerical simulations for $D_{v} / D_{u}=100$ because the approximation to obtain the reduced system (7) becomes better for larger values of $D_{v} / D_{u}$. However, this situation is not easily accessible experimentally. Therefore we here describe the results for smaller value of $D_{v} / D_{u}$. We have carried out numerical simulations for $D_{u}$ $=5.0 \times 10^{-5}, D_{v}=5.0 \times 10^{-4}, \alpha=0.5$, and $\gamma=2.5$ and changing the parameter $\beta$ and the cell size $\delta x$. The cell size is changed in the same way as mentioned above. The asymptotic stationary solutions obtained are summarized in Fig. 10. This was obtained starting from different random initial conditions for a given value of $\beta$ and a given cell size $\delta x$. Note that four or more different structures appear for the

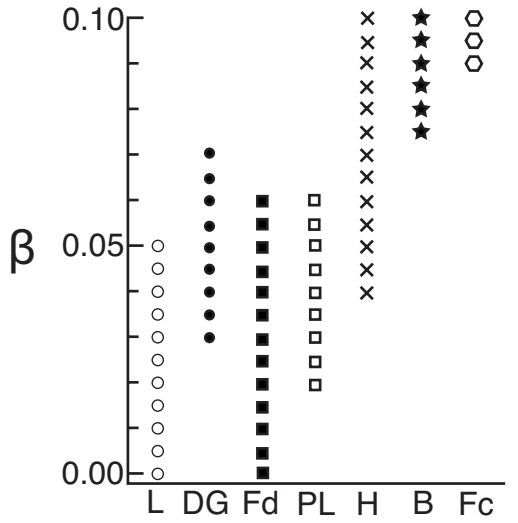

FIG. 10. Seven stationary solutions for $D_{v} / D_{u}=10.0$ in the FitzHugh-Nagumo equation. The meanings of L, DG, Fd, PL, H, B, and $\mathrm{Fc}$ are the same as those in Fig. 1. It is noted that we have not obtained SG and SD in this ratio of the diffusion constants.

given set of parameters. It is obvious that the existence of the structures has the same tendency as the one in Fig. 1.

\section{BRUSSELATOR}

In Sec. II, we have shown that interconnected periodic patterns are self-organized in the FitzHugh-Nagumo equation in three dimensions. In order to confirm that this result is not restricted to the FitzHugh-Nagumo system, we here study the Brusselator [13] given by the set of equations

$$
\begin{gathered}
\frac{\partial u}{\partial t}=D_{u} \nabla^{2} u+A-(B+1) u+u^{2} v, \\
\frac{\partial v}{\partial t}=D_{v} \nabla^{2} v+B u-u^{2} v,
\end{gathered}
$$

where $A$ and $B$ are positive parameters and $D_{u}$ and $D_{v}$ are diffusion constants.

The equilibrium stable uniform solution of Eqs. (16) and (17) is given by $u=u_{0}=A$ and $v=v_{0}=B / A$ as the Brusselator is always monostable. Putting $\delta u=u-u_{0}$ and $\delta v=v-v_{0}$, Eqs. (16) and (17) can be written as

$$
\begin{gathered}
\frac{\partial \delta u}{\partial t}=\left(D_{u} \nabla^{2}+B-1\right) \delta u+A^{2} \delta v+g(\delta u, \delta v), \\
\frac{\partial \delta v}{\partial t}=-B \delta u+\left(D_{v} \nabla^{2}-A^{2}\right) \delta v-g(\delta u, \delta v),
\end{gathered}
$$

where $g(\delta u, \delta v)=2 A \delta u \delta v+(B / A) \delta u^{2}+\delta u^{2} \delta v$. It is readily shown that the uniform time-independent solution is linearly unstable if the eigenvalue $\lambda$ given by the solution of the equation

$$
\lambda^{2}+\alpha_{k} \lambda+\beta_{k}=0
$$

is positive, where

$$
\alpha_{k}=\left(D_{u}+D_{v}\right) k^{2}+A^{2}-B+1,
$$


TABLE VI. The representative values of the system size $L$ $=\delta x \times N$ with the cell size $\delta x$ and the noise strength $\sigma$, which produce the stationary solutions in the Brusselator for $B=4.0$ and the cell number $N=32$.

\begin{tabular}{lccc}
\hline \hline Pattern & $L$ & $\delta x$ & $\sigma$ \\
\hline L & 0.128 & 0.00400 & $5.0 \times 10^{-3}$ \\
SG & 0.124 & 0.00388 & $1.0 \times 10^{-2}$ \\
SD & 0.160 & 0.00500 & $5.0 \times 10^{-3}$ \\
DG & 0.208 & 0.00650 & $1.0 \times 10^{-2}$ \\
Fd & 0.212 & 0.00663 & $5.0 \times 10^{-3}$ \\
PL & 0.164 & 0.00513 & $1.0 \times 10^{-3}$ \\
H & 0.230 & 0.00719 & $1.0 \times 10^{-3}$ \\
\hline \hline
\end{tabular}

$$
\beta_{k}=D_{u} D_{v} k^{4}+\left(D_{u} A^{2}+D_{v}-D_{v} B\right) k^{2}+A^{2},
$$

with $k$ the wave number of spatial modulation. Turing instability occurs for $B>B_{c}$ where $B_{c}=\left[1+A\left(D_{u} / D_{v}\right)^{1 / 2}\right]^{2}$ with the critical wave number given by $k_{c}^{2}=A /\left(D_{u} D_{v}\right)^{1 / 2}$. In the Appendix, we derive approximately a variational reduced system of the Brusselator near the Turing instability threshold, which is similar to the one given by Eq. (7) with Eq. (8).

Three-dimensional Turing patterns in the Brusselator have been studied both numerically and analytically [20,22,23]. De Wit et al. considered the possibilities of the formation of periodic minimal surfaces for the parameters $D_{u}=1, D_{v}=8$, and $A=4.5$ [22]. In this set of parameters the threshold value is given by $B_{c}=6.713$. By changing the value of $B$ up to $B$ $=8.2$, however, they did not find any clear periodic interconnected patterns. We have also tried numerical simulations up to $B=11$. It has been verified that a double-gyroid structure is stable in the sense that it does not break down under the random disturbances if the gyroid is provided initially. However, we have not succeeded in producing it spontaneously starting from random initial concentrations as was done for the FitzHugh-Nagumo equation in the previous section. One of the reasons is that the condition $B \cong 10$ is fairly far away from the Turing bifurcation threshold so that the interface

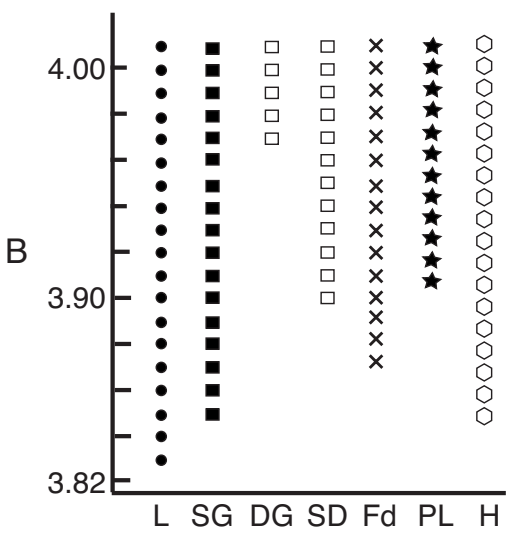

FIG. 11. Seven stationary solutions for the Brusselator. The meanings of L, DG, SG, SD, Fd, PL, and $\mathrm{H}$ are the same as those in Fig. 1. bcc and fec structures exist for larger values of $B$ as mentioned in the text.

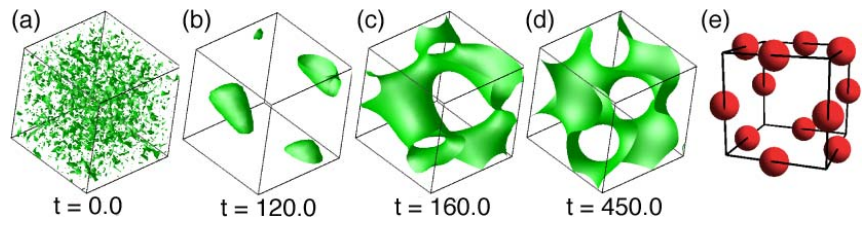

FIG. 12. (Color online) Single-gyroid structure obtained in the Brusselator (a)-(d) Structural evolution of domains for $B=4.00, L$ $=0.124=\delta x N$ with $\delta x=3.88 \times 10^{-3}$ and $N=32$, and $\sigma=1.0 \times 10^{-2}$, and (e) Bragg spots for $I_{k}>1.5 \times 10^{-4}$. The meanings of (e) are the same as those in Fig. 2. In order to make the initial randomness visible the domains in (a) represent the isosurface of $u=2.7002$ whereas those in (b)-(d) represent the isosurface of $u=2.7$.

separating two domains is quite sharp and therefore it is difficult to reach the true stationary solutions before the patterns get trapped in a metastable state.

In order to avoid the difficulty mentioned above, we have carried out numerical simulations for different values of the parameters. That is, we have chosen as $D_{u}=2.0 \times 10^{-4}, D_{v}$ $=1.6 \times 10^{-3}$, and $A=2.7$ and changing the parameter $B$ and the cell size $\delta x$. The value of $B$ at the bifurcation threshold is given, in this case, by $B_{c}=3.82$. We explore the region for $B \leqslant 4.02$ rather close to the bifurcation threshold. The calculation scheme is the same as the one in the previous section. We start with the unstable uniform solution $(\bar{u}, \bar{v})$ randomly disturbed. Uncorrelated random forces are added to Eqs. (16) and (17) in the simulations.

The asymptotic stationary solutions obtained are summarized in Fig. 11. This diagram was obtained by five independent runs for a given value of $B$ and for a given cell size. Note that three or more different structures coexist. The abbreviations L, H, DG, Fd, PL, SG, and SD are the same as those in Fig. 1. That is, all the patterns found in the FitzHugh-Nagumo equation appear also in the Brusselator. (fcc and bcc structures will be mentioned in the final part of this section.) The representative values of the system size $L$, the cell size $\delta x$, and the noise strength $\sigma$ for the patterns in Fig. 11 are listed in Table VI.

The time-evolution of the structure of SG and the Bragg spots are shown in Fig. 12. The Bragg peaks are listed together with the peak intensities in Table VII. The peak positions are similar to those of bcc (Table IV). This network structure is called a single-gyroid structure, which is one of the structures composed of minimal surfaces.

The formation of SD and the Bragg positions are shown in Fig. 13. The Bragg peaks are listed together with the peak intensities in Table VIII. The peak positions are similar to those of FCC (Table V). This network structure is called a single diamond structure.

TABLE VII. Bragg spots and their intensities (arbitrary unit) of single-gyroid structure.

\begin{tabular}{lccc}
\hline \hline Peak position & $I_{k}$ & Peak position & $I_{k}$ \\
\hline$(1,1,0)$ & 0.064480 & $(1,-1,0)$ & 0.064614 \\
$(1,0,1)$ & 0.064858 & $(0,1,1)$ & 0.064740 \\
$(1,0,-1)$ & 0.064506 & $(0,1,-1)$ & 0.064958 \\
\hline \hline
\end{tabular}


Finally we remark about fcc and bcc structures. When $B$ is smaller than 4.02, these structures are not obtained. However, we have verified numerically the existence of these structures for larger values of $B$. For instance, we have fcc structure for $B=6.0, L=0.176$ with $\delta x=5.49 \times 10^{-3}$ and $N$ $=32$, and $\sigma=8.0 \times 10^{-2}$, whereas bcc structure appears for $B=6.0, L=0.1076$ with $\delta x=3.36 \times 10^{-3}$ and $N=32$, and $\sigma$ $=8.0 \times 10^{-3}$. (These results are not shown.)

\section{GRAY-SCOTT MODEL}

As the third model we study the Gray-Scott model [27] to confirm that interconnected periodic patterns exist as a Turing pattern. The Gray-Scott model describes the chemical reactions $U+2 V \rightarrow 3 V$ and $V \rightarrow W$ where $W$ is an inert product. The equations for the concentrations of the two chemicals $u$ and $v$ are given in dimensionless units by

$$
\begin{aligned}
& \frac{\partial u}{\partial t}=D_{u} \nabla^{2} u-u v^{2}+F(1-u), \\
& \frac{\partial v}{\partial t}=D_{v} \nabla^{2} v+u v^{2}-(F+K) v,
\end{aligned}
$$

where $D_{u}$ and $D_{v}$ are the diffusion constants. The constant $F$ is the in-flow rate of $U$ from the outside and $F+K$ is the removal rate of $V$ from the reaction field.

Pearson [44] studied the Gray-Scott model numerically in two dimensions. This model exhibits a very rich behavior ranging from time-independent steady solutions to chaotic, oscillatory, and time-dependent phase turbulent behavior. Leppanen et al. [21] studied the Gray-Scott model numerically in three dimensions. However, they did not obtain any clear evidence of the formation of periodic interconnected patterns.

In the present paper, numerical simulations are performed for $D_{u}=2.0 \times 10^{-4}$ and $D_{v}=1.0 \times 10^{-4}$ and choosing a different cell size $\delta x$ in each run. We set $K=0.062$ and change $F$ such that the system is monostable without diffusion. The stable solution is given by $u=1$ and $v=0$. The main reason as to why we choose this set of parameters is that Pearson [44] has found a self-replication of spots in two dimensions and an asymptotic hexagonal structure of these spots. It is interesting to see what happens in three dimensions. It should be mentioned here that the amplitude of the Turing patterns obtained below is small since the system is close to the bifurcation threshold.

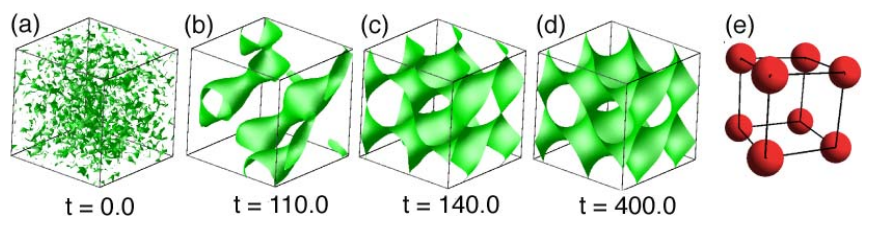

FIG. 13. (Color online) Single-diamond structure obtained in the Brusselator. (a)-(d) Structural evolution of domains for $B=4.00$, $L=0.160=\delta x N$ with $\delta x=5.00 \times 10^{-3}$ and $N=32$, and $\sigma=5.0 \times 10^{-3}$, and (e) Bragg spots for $I_{k}>10^{-4}$. The meanings of (e) are the same as those in Fig. 2. The values of the isosurface are the same as those in Fig. 12.
TABLE VIII. Bragg spots and their intensities (arbitrary unit) of single-diamond structure.

\begin{tabular}{lccc}
\hline \hline Peak position & $I_{k}$ & Peak position & $I_{k}$ \\
\hline$(1,1,1)$ & 0.058675 & $(1,-1,1)$ & 0.058850 \\
$(1,1,-1)$ & 0.063469 & $(1,-1,-1)$ & 0.057721 \\
\hline \hline
\end{tabular}

Following the method in Ref. [44], the initial concentrations of $u$ and $v$ are provided such that $u=1$ and $v=0$ everywhere except for the cubic region with the linear dimension $10 \delta x$ at the center of the system where $u=0.5$ and $v=0.25$ with small random perturbations. Random forces are added to Eqs. (23) and (24) in the simulations.

The formation of double-gyroid structure obtained in the Gray-Scott model is displayed in Fig. 14. The initial cubic area expands as shown in Figs. 14(b) and 14(c). After the entire system is covered by the expanded domain, the domain starts to change shape as in Fig. 14(d). Many voids develop gradually in Figs. $14(\mathrm{e})-14(\mathrm{~g})$ to constitute an asymptotic double-gyroid pattern in Fig. 14(h).

The time evolution of other patterns listed in Table IX obtained from the same initial conditions is qualitatively the same as the above. The representative values of the parameter $F$, the system size $L$, the mesh size $\delta x$, and the noise strength $\sigma$ are given in Table IX. It should be noted that we have not found single-gyroid and single-diamond structures in the Gray-Scott model in contrast to the cases of the FitzHugh-Nagumo equation and the Brusselator.

\section{DISCUSSION}

In this paper, we have studied Turing patterns numerically in the FitzHugh-Nagumo equation, the Brusselator, and the Gray-Scott model in three dimensions. Apart from lamellar and hexagonal structures which are a simple generalization of two-dimensional patterns, we have obtained doublegyroid, Fddd, and perforated-lamellar structures. Furthermore, single-gyroid structure and single-diamond structure are also obtained for the FitzHugh-Nagumo equation and the
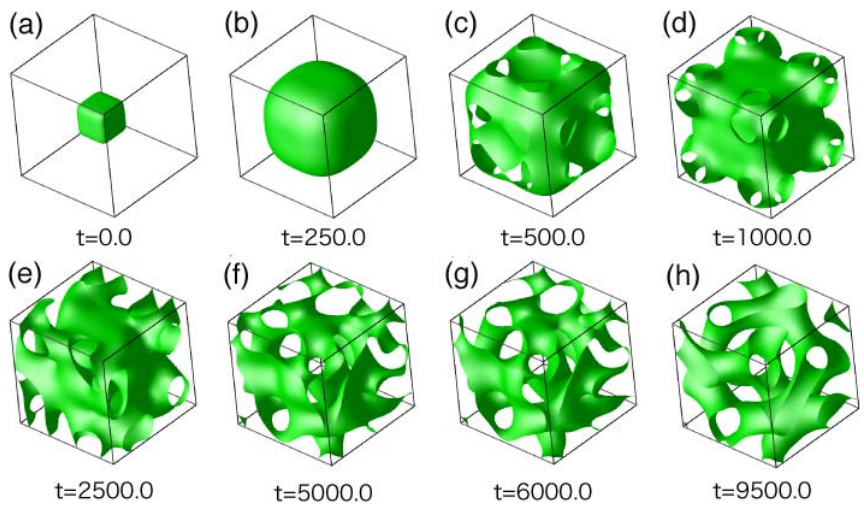

FIG. 14. (Color online) (a)-(h) Formation of double-gyroid structure in the Gray-Scott model for $F=0.03 L=1.10=\delta x N$ with $\delta x=0.0344$ and $N=32$, and $\sigma=1.0 \times 10^{-3}$. (a)-(h) represent the isosurface of $u=0.55$. 
TABLE IX. Seven stationary solutions for the Gray-Scott model for $N=32$ and the representative values of the parameter $F$, the system size $L=\delta x \times N$ with the cell size $\delta x$, and the strength $\sigma$ of noises. The meanings of L, H, PL, Fc, DG, and Fd are the same as those in Fig. 1.

\begin{tabular}{lcccc}
\hline \hline Pattern & $F$ & $L$ & $\delta x$ & $\sigma$ \\
\hline L & 0.030 & 1.120 & 0.035 & $1.0 \times 10^{-3}$ \\
H & 0.025 & 1.216 & 0.038 & $1.0 \times 10^{-3}$ \\
PL & 0.027 & 1.218 & 0.038 & $2.0 \times 10^{-4}$ \\
Fc & 0.022 & 0.896 & 0.028 & $1.0 \times 10^{-3}$ \\
B & 0.022 & 1.472 & 0.046 & $5.0 \times 10^{-4}$ \\
DG & 0.030 & 1.088 & 0.034 & $1.0 \times 10^{-3}$ \\
Fd & 0.030 & 1.086 & 0.034 & $9.0 \times 10^{-4}$ \\
\hline \hline
\end{tabular}

Brusselator. These five patterns are interconnected periodic structures and inherently three-dimensional structures.

Double-gyroid, single-gyroid, and single-diamond structures are known as a periodic minimal surface where the average curvature is equal to zero everywhere on the surface [24]. There are two other periodic minimal surfaces, doublediamond and Schwarz P surfaces. The P surface structure has not been observed as a stationary solution in our present simulations. It should be mentioned, however, that the P surface structure appears as a transient solution in the Brusselator for $B=3.825, L=0.0909=\delta x N$ with $\delta x=2.84 \times 10^{-3}$ and $N=32$, and $\sigma=1.0 \times 10^{-5}$, although it is changed to a lamellar structure eventually. In the present simulations we have never observed double-diamond structure.

We have found that both bcc and fcc structures constituted by disconnected spherical domains are the stationary solutions in the three model systems studied here. However, it is mentioned that a simple cubic structure has not been obtained as a stationary solution but has been observed transiently during the process of the formation of fcc structure in the FitzHugh-Nagumo equation for $\beta=0.09, L=0.142=\delta x N$ with $\delta x=4.43 \times 10^{-3}$ and $N=32$, and $\sigma=1.0 \times 10^{-5}$.

In the present paper, we have chosen a system size such that it is just one spatial period of the structures. This is mainly because numerical simulations in a larger system size are time consuming in three dimensions and are inaccessible by our computer facilities. We have, of course, noticed that the system size often plays a crucial role for the stability of the periodic solutions [45-47]. Therefore, we have partly verified the stability by carrying out simulations of the Brusselator for a larger system having the size twice of the spatial period. For example, single gyroid for $B=3.845, L=0.2556$ $=\delta x N$ with $\delta x=7.9875 \times 10^{-3}$ and $N=32$, and $\sigma=1.0 \times 10^{-4}$ and single diamond for $B=3.9, L=0.3088=\delta x N$ with $\delta x$ $=9.65 \times 10^{-3}$ and $N=32$, and $\sigma=1.0 \times 10^{-4}$ can appear in this larger system in complete accordance with the smaller system.

Although we have not succeeded in obtaining the doublegyroid structure in a larger system in the Brusselator as well as in the other two reaction diffusion systems, we have checked the stability in several alternative ways. First of all, the double-gyroid structure takes the minimum value of the free energy functional (8) in the reduced system as shown in
TABLE X. Turing patterns obtained by the present study. FHN, BRS, and GS mean the FitzHugh-Nagumo equation, the Brusselator, and the Gray-Scott model, respectively. The patterns indicated by $\odot$ were obtained by the numerical schemes of the libfles_fft library [33] and by the forward Euler integration method. The patterns indicated by $\bigcirc$ were obtained only by the scheme of the libfles_fft library. The structures marked by $*$ have been obtained as thermally equilibrium ones in diblock copolymers [37,41,43]. Double diamond (DD) and P (Schwarz P surface) have not been found in the present simulations.

\begin{tabular}{lccccccccccc}
\hline \hline & $\mathrm{L}^{*}$ & $\mathrm{H}^{*}$ & $\mathrm{PL}$ & $\mathrm{B}^{*}$ & $\mathrm{Fc}$ & $\mathrm{DG}^{*}$ & $\mathrm{SG}$ & $\mathrm{DD}$ & $\mathrm{SD}$ & $\mathrm{P}$ & $\mathrm{Fd}^{*}$ \\
\hline $\mathrm{FHN}$ & $\odot$ & $\odot$ & $\odot$ & $\odot$ & $\odot$ & $\odot$ & $\odot$ & & $\odot$ & & $\odot$ \\
$\mathrm{BRS}$ & $\odot$ & $\odot$ & $\odot$ & $\odot$ & $\odot$ & $\odot$ & $\bigcirc$ & & $\bigcirc$ & \\
$\mathrm{GS}$ & $\odot$ & $\bigcirc$ & $\bigcirc$ & $\bigcirc$ & $\bigcirc$ & $\bigcirc$ & & & & & $\bigcirc$ \\
\hline \hline
\end{tabular}

the phase diagram, Fig. 8. This analysis is independent of the system size and confirms the stability of the double-gyroid structure at least in the situation that the variable $v$ can be eliminated adiabatically. In fact, the structure formation along the line $\gamma=26$ is qualitatively consistent with that of Fig. 1. Second, in our previous paper [25], we examined the robustness of the structures by increasing the noise strength. It was found that the double-gyroid structure is mostly stable among other structures-e.g., for $\beta=0.04$ in the FitzHughNagumo equation. These are indeed evidence of the structural stability of the numerical results. Third, we have applied the numerical scheme carefully to eliminate the anisotropy due to the cubic cells. We admit, however, that simulations by means of another algorithm such as the finiteelement method—e.g., [48]—which does not rely on the cubic cells, are necessary since gyroid structure itself has a cubic symmetry. However, this is beyond the scope of the present study.

We shall discuss the numerical methods a little bit more. In order to verify that the obtained structures are independent of the numerical scheme we have employed two methods of simulations. One is the forward Euler integrations of the finite-difference equations, and the other is the so-called libfles_fft library method [33]. As is seen from Table X, all the patterns except for single-diamond and single-gyroid structures are obtained in the FitzHugh-Nagumo equation and in the Brusselator by both methods. In the case of the Gray-Scott model, we have not applied both methods systematically because it takes too much time. It should be noted that the forward Euler integration method requires smaller time increments to get sufficient accuracy and to avoid numerical instabilities.

The time to reach the asymptotic patterns depends on various factors. It is noted that there are generally two stages in the domain evolution starting from the random initial state. At the early stage, the exponential growth of the unstable modes occurs. This time interval is insensitive to the final structure. The second stage is the nonlinear evolution of domains. This second period strongly depends on the evolving structure. As can be seen from Figs. 2, 6, 7, 12, and 13, the structures having a cubic symmetry reach the final pattern at about $t \cong 500-600$. However, the modulated uniaxial 
structures PL and Fddd take a longer time $t \cong 750-850$ as is shown in Figs. 4 and 5. Simpler disconnected structures such as a hexagonal structure grow more rapidly $t \cong 100$ (data are not shown).

From a technical viewpoint, the evolution time depends drastically on the system size, the numerical scheme, and so forth. If there is an even small discrepancy between the system size and the true spatial period of the final structure, the time to achieve the final structure becomes substantially longer. Comparing the two schemes of simulations, the forward Euler integration method takes a longer time to reach the asymptotic state than the libfles_fft library.

One of the most important conclusions of the present paper is that periodic minimal surface structures appear in three different reaction diffusion systems as summarized in Table $X$. It is quite interesting to see that similar interconnected structures have been observed in soft condensed matter in thermal equilibrium $[24,49]$. This can be understood partly as we have shown in Sec. II that the FitzHugh-Nagumo equation has a Lyapunov functional in some special limit as Eqs. (7) and (8) which is the same as the free energy functional of diblock copolymers. However, it should be noted from Table $\mathrm{X}$ that Turing patterns contain more varieties of structures compared to microphase-separated structures indicated by an asterisk $(*)$ in Table X. There are several reasons for this. First of all, the long-range interaction in diblock copolymer melts is just Coulomb type [40] whereas the long-range interaction in Eq. (8) is the Yukawa type with screening. Second, there is a concentration conservation in microphase separation, but no such conservation in reaction diffusion systems. Finally, the original (not reduced one) reaction diffusion equations studied here are nonvariational. We expect that these facts cause less restricted conditions for pattern formation far from equilibrium.

\section{ACKNOWLEDGMENTS}

We would like to thank Professor R. Kobayashi for valuable discussions and useful advice in numerical simulations. H.S. and K.Y. are supported by a Grant-in-Aid from the Japan Society for the Promotion of Science. This work was supported in part by a Grant-in-Aid for the 21st Century COE "Center for Diversity and Universality in Physics" and the Grant-in-Aid for the superior area "Soft Matter Physics," both from the Ministry of Education, Culture, Sports, Science and Technology (MEXT) of Japan.

\section{APPENDIX}

Here we derive an approximate reduced system for Brusselator near the Turing bifurcation threshold. The solution of Eq. (20) is obtained as

$$
\lambda_{ \pm}=-\frac{1}{2} \alpha_{k} \pm \frac{1}{2} \sqrt{\alpha_{k}^{2}-4 \beta_{k}},
$$

where $\lambda_{-}<0$, but $\lambda_{+}>0$ at post-threshold and hence the mode with $\lambda_{-}<0$ is irrelevant. The corresponding eigenvectors are readily calculated, which take the form of $\left(A^{2}, \gamma_{ \pm}\right)$ with

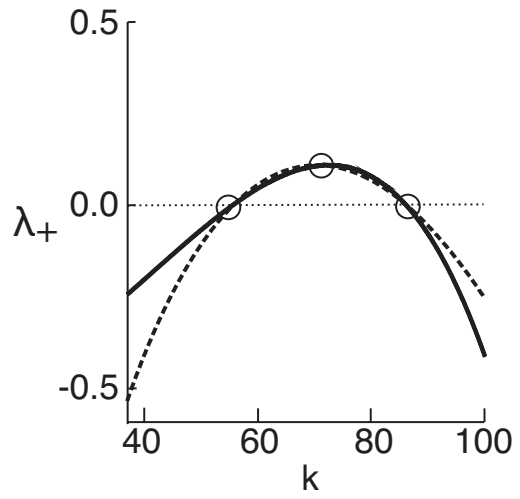

FIG. 15. Approximant for $\lambda_{+}$. The dotted line is given by Eq. (A1) for $D_{u}=2.0 \times 10^{-4}, D_{v}=1.6 \times 10^{-3}, A=2.7$, and $B=4.0$. The solid line is the approximant given by Eq. (A9).

$$
\gamma_{ \pm}=\frac{1}{2}\left(D_{u}-D_{v}\right) k^{2}-\frac{1}{2}\left(A^{2}+B-1\right) \pm \frac{1}{2} \sqrt{\alpha_{k}^{2}-4 \beta_{k}} .
$$

Let us introduce

$$
\begin{gathered}
w=-\frac{1}{A^{2} \sqrt{\alpha_{k}^{2}-4 \beta_{k}}}\left(\gamma_{-} x-A^{2} y\right), \\
w^{\prime}=-\frac{1}{A^{2} \sqrt{\alpha_{k}^{2}-4 \beta_{k}}}\left(-\gamma_{+} x+A^{2} y\right),
\end{gathered}
$$

and substitute them into Eqs. (16) and (17) so that we obtain

$$
\frac{\partial w}{\partial t}=\lambda_{+} w+N_{w},
$$

$$
\frac{\partial w^{\prime}}{\partial t}=\lambda \_w^{\prime}+N_{w^{\prime}},
$$

where

$$
\begin{aligned}
N_{w}= & -\frac{\gamma_{-}+A^{2}}{\sqrt{\alpha_{k}^{2}-4 \beta_{k}}}\left[A B\left(w+w^{\prime}\right)^{2}+2 A\left(w+w^{\prime}\right)\left(\gamma_{+} w+\gamma_{-} w^{\prime}\right)\right. \\
& \left.+A^{2}\left(w+w^{\prime}\right)^{2}\left(\gamma_{+} w+\gamma_{-} w^{\prime}\right)\right]
\end{aligned}
$$

and

$$
\begin{aligned}
N_{w^{\prime}}= & \frac{\gamma_{+}+A^{2}}{\sqrt{\alpha_{k}^{2}-4 \beta_{k}}}\left[A B\left(w+w^{\prime}\right)^{2}+2 A\left(w+w^{\prime}\right)\left(\gamma_{+} w+\gamma_{-} w^{\prime}\right)\right. \\
& \left.+A^{2}\left(w+w^{\prime}\right)^{2}\left(\gamma_{+} w+\gamma_{-} w^{\prime}\right)\right] .
\end{aligned}
$$

By taking account of the three points indicated in Fig. 15, the eigenvalue of the unstable mode $\lambda_{+}$is expressed approximately as

$$
\lambda_{+} \cong-Q k^{2}-\frac{P}{k^{2}+c_{1}}+c_{2},
$$

where $P, Q, c_{1}$, and $c_{2}$ are constants depending on $A, B, D_{u}$, and $D_{v} . P$ and $Q$ should not be confused with the magnitude of the wave numbers introduced in Sec. II. On the other 
hand, the eigenvalue $\lambda_{-}$of the stable mode can be approximated near the Turing bifurcation threshold as

$$
\lambda_{-}=-A\left(A+\sqrt{\frac{D_{v}}{D_{u}}}\right)\left(1-\frac{D_{u}}{D_{v}}\right) .
$$

Since $\left|\lambda_{-}\right| \gg\left|\lambda_{+}\right|$near the bifurcation threshold, we may eliminate the stable mode $w^{\prime}$ adiabatically by putting $\partial w^{\prime} / \partial t=0$ in Eq. (A6):

$$
\begin{aligned}
w^{\prime} \cong & -\frac{A B+2 A \gamma_{+}}{\frac{\sqrt{\alpha_{k}^{2}-4 \beta_{k}}}{\gamma_{+}+A^{2}} \lambda_{-}+2 A \gamma_{+}+2 A \gamma_{-}} w^{2} \\
& -\left[\frac{A^{2} \gamma_{+}}{\frac{\sqrt{\alpha_{k}^{2}-4 \beta_{k}}}{\gamma_{+}+A^{2}} \lambda_{-}+2 A \gamma_{+}+2 A \gamma_{-}}\right. \\
& \left.-\frac{2 A^{2} B^{2}}{\left(\frac{\sqrt{\alpha_{k}^{2}-4 \beta_{k}}}{\gamma_{+}+A^{2}} \lambda_{-}+2 A \gamma_{+}+2 A \gamma_{-}\right)^{2}}\right] w^{3} .
\end{aligned}
$$

Here $w^{\prime}$ is obtained as a power of $w$ which is valid near the bifurcation where $w$ is small. Furthermore, we use the following approximation under the condition $\left|\beta_{k}\right| \ll 1$ :

$$
\sqrt{\alpha_{k}^{2}-4 \beta_{k}} \cong \alpha_{k_{c}}
$$

Using the relation $B_{c}=\left(1+\sqrt{D_{u} / D_{v}} A\right)^{2}$ for the Turing bifurcation point $B_{c}$ we obtain $\gamma_{+}=D_{u} k_{c}^{2}-B_{c}+1$ and $\gamma_{-}=-D_{v} k_{c}^{2}$ $-A^{2}$. Substituting Eq. (A11) into Eq. (A7) and expanding it in terms of $w$ we obtain up to order of $w^{3}$

$$
\begin{aligned}
N_{w} \cong & -\frac{\gamma_{-}+A^{2}}{\alpha_{k_{c}}}\left(A B+2 A \gamma_{+}\right) w^{2}+\frac{\gamma_{-}+A^{2}}{\alpha_{k_{c}}} \\
& \times\left[-A^{2} \gamma_{+}+\frac{\left(2 A B+2 A \gamma_{+}+2 A \gamma_{-}\right)\left(A B+2 A \gamma_{+}\right)}{\frac{\alpha_{k_{c}}}{\gamma_{+}+A^{2}} \lambda_{-}+2 A \gamma_{+}+2 A \gamma_{-}}\right] w^{3} .
\end{aligned}
$$

Substituting this into Eq. (A5) we obtain

$$
\frac{\partial w}{\partial t}=\left[\lambda_{+} w+a_{3} w^{2}+a_{4} w^{3}\right]
$$

where the coefficeints $a_{3}$ and $a_{4}(<0)$ depend on $A, B, D_{u}$, and $D_{v}$.

Notice that Eq. (A14) with Eq. (A9) can be written as

$$
\frac{\partial w}{\partial t}=-\frac{\delta H}{\delta w},
$$

where

$$
\begin{aligned}
H\{w\}= & \int d \vec{r}\left[\frac{D}{2}(\nabla w)^{2}-\frac{a_{2}}{2} w^{2}-\frac{a_{3}}{3} w^{3}-\frac{a_{4}}{4} w^{4}\right] \\
& +\frac{K}{2} \int d \vec{r} \int d \vec{r}^{\prime} G\left(\vec{r}, \vec{r}^{\prime}\right) w(\vec{r}) w\left(\vec{r}^{\prime}\right) .
\end{aligned}
$$

The constants $D, K$, and $a_{2}$ are given by $D=Q, K=P$, and $a_{2}=c_{2}$, respectively. The Green function $G(\vec{r}, \vec{r})$ is defined through the relation

$$
\left(-\nabla^{2}+c_{1}\right) G\left(\vec{r}, \vec{r}^{\prime}\right)=\delta\left(\vec{r}-\vec{r}^{\prime}\right) .
$$

$H\{w\}$ plays a role of a Lyapunov functional as the case of Eq. (7) in the FitzHugh-Nagumo equation.

Here we make a remark that Eq. (A16) has an asymmetric term $w^{3}$ and that the coefficient $a_{3}$ can be shown to increase monotoneously as the value of the parameter $B$ is increased.
[1] G. Nicolis and I. Prigogine, Self-Organization in Nonequilibrium Systems (Wiley, New York, 1977).

[2] Y. Kuramoto, Chemical Oscillation, Waves and Turbulance (Springer-Verlag, Berlin, 1984).

[3] Wave and Patterns in Biological and Chemical Excitable Media, edited by V. Krinsky and H. Swinney (North-Holland, Amsterdam, 1991).

[4] M. C. Cross and P. C. Hohenberg, Rev. Mod. Phys. 65, 851 (1993).

[5] J. D. Murray, Mathematical Biology, 2nd ed. (Springer-Verlag, Berlin, 1993)

[6] A. M. Turing, Philos. Trans. R. Soc. London, Ser. B 237, 37 (1952).

[7] V. Castets, E. Dulos, J. Boissonade, and P. De Kepper, Phys. Rev. Lett. 64, 2953 (1990).

[8] P. De Kepper, V. Castets, E. Dulos, and J. Boissonade, Physica D 49, 161 (1991).

[9] Q. Ouyang and H. L. Swinney, Nature (London) 352, 610 (1991).
[10] Q. Ouyang and H. L. Swinney, Chaos 1, 411 (1991).

[11] J. J. Perraud, K. Agladze, E. Dulos, and P. De Kepper, Physica A 188, 1 (1992).

[12] Q. Ouyang, Z. Noszticzius, and H. L. Swinney, J. Phys. Chem. 96, 6773 (1992).

[13] I. Prigogine and R. Lefever, J. Chem. Phys. 48, 1695 (1968); I. Prigogine, R. Lefever, A. Goldbeter, and M. HerschkowitzKaufman, Nature (London) 223, 913 (1969).

[14] D. Walgraef, G. Dewel, and P. Borckmans, Phys. Rev. A 21, 397 (1980).

[15] E. Dulos, P. Davies, B. Rudovics, and P. De Kepper, Physica D 98, 53 (1996).

[16] S. Setayeshgar and M. C. Cross, Phys. Rev. E 58, 4485 (1998); 59, 4258 (1999).

[17] I. R. Epstein, I. Lengyel, S. Kadar, M. Kagan, and M. Yokoyama, Physica A 188, 26 (1992).

[18] G. Dewel, M. Bachir, S. Metens, and P. Borckmans, Faraday Discuss. 120, 363 (2001).

[19] L. Yang, M. Dolnik, A. M. Zhabotinsky, and I. R. Epstein, 
Phys. Rev. Lett. 88, 208303 (2002).

[20] A. De Wit, G. Dewel, P. Borckmans, and D. Walgraef, Physica D 61, 289 (1992).

[21] T. Leppanen, M. Karttunen, K. Kaski, R. A. Barrio, and L. Zhang, Physica D 168, 35 (2002); T. Leppanen, M. Karttunen, R. A. Barrio, and K. Kaski, Phys. Rev. E 70, 066202 (2004); Braz. J. Phys. 34, 368 (2004).

[22] A. De Wit, P. Borckmans, and G. Dewel, Proc. Natl. Acad. Sci. U.S.A. 94, 12765 (1997).

[23] T. Callahan and E. Knobloch, Physica D 132, 339 (1999) and earlier references.

[24] Morphology of Condensed Matter: Physics and Geometry of Spatially Complex Systems, edited by Klaus R. Mecke and Dietrich Stoyan (Springer, New York, 2003).

[25] H. Shoji, K. Yamada, and T. Ohta, Phys. Rev. E 72, 065202(R) (2005).

[26] R. FitzHugh, Biophys. J. 1, 445 (1961); J. Nagumo, S. Arimoto, and S. Yoshizawa, Proc. IRE 50, 2061 (1962).

[27] P. Gray and S. K. Scott, Chem. Eng. Sci. 38, 29 (1983).

[28] J. Rinzel and J. B. Keller, Biophys. J. 13, 1313 (1973).

[29] J. J. Tyson and J. P. Keener, Physica D 32, 327 (1988).

[30] J. Boissonade and P. De Kepper, J. Phys. Chem. 84, 501 (1980).

[31] A. Malevanets and R. Kapral, Phys. Rev. E 55, 5657 (1997).

[32] S. Metens, G. Dewel, P. Borckmans, and R. Engelhardt, Europhys. Lett. 37, 109 (1997).

[33] M. Nonomura, D. Ueyama, and R. Kobayashi (unpublished).

[34] D. J. Eyre, in Computational and Mathematical Models of Microstructural Evolution, edited by J. W. Bullard, R. Kalia, M. Stoneham, and L-Q. Chen, MRS symposia No. 529 (Materials
Research Society, San Francisco, 1998).

[35] A. Shinozaki and Y. Oono, Phys. Rev. E 48, 2622 (1993).

[36] A. Aksimetiev, M. Fialkowski, and R. Holyst, Adv. Chem. Phys. 121, 141 (2002).

[37] C. A. Tyler and D. C. Morse, Phys. Rev. Lett. 94, 208302 (2005).

[38] K. Yamada, M. Nonomura, and T. Ohta, J. Phys.: Condens. Matter 18, L421 (2006).

[39] T. Ohta, Prog. Theor. Phys. Suppl. 99, 425 (1989).

[40] T. Ohta and K. Kawasaki, Macromolecules 19, 2621 (1986).

[41] T. Teramoto and Y. Nishiura, J. Phys. Soc. Jpn. 71, 1611 (2002).

[42] T. Garel and S. Doniach, Phys. Rev. B 26, 325 (1982); D. Andelman, F. Brochard, and J. F. Joanny, J. Chem. Phys. 86, 3673 (1987); M. Hoshino, H. Nakano, and H. Kimura, J. Phys. Soc. Jpn. 46, 1709 (1979).

[43] K. Yamada, M. Nonomura, and T. Ohta, Macromolecules 37, 5762 (2004).

[44] J. E. Pearson, Science 261, 189 (1993).

[45] T. Ohta, M. Mimura, and R. Kobayashi, Physica D 34, 115 (1989).

[46] A. L. Kawczynski and B. Legawiec, Phys. Rev. E 63, 021405 (2001).

[47] A. L. Kawczynski and M. Leda, Phys. Rev. E 73, 056208 (2006).

[48] M. Kimura, H. Komura, M. Mimura, H. Miyoshi, T. Takaishi, and D. Ueyama (unpublished).

[49] Bicontinuous Liquid Crystals, edited by M. L. Lynch and P. T. Spicer (Taylor \& Francis, New York, 2005). 